\title{
UNE STÈLE SUR LE PARVIS DU TEMPLE D’OPET À KARNAK
}

\author{
[Planches I-VI]
}

PAR

\section{Laurent COULON}

Université de Lyon-II/CNRS HISOMA UMR 5189

Maison de l'Orient et de la Méditerranée

$5 / 7$ rue Raulin

F-69007 Lyon

\author{
Luc GABOLDE
}

(CNRS -

FRE 2742)

2, rue d'Oran

F-69001 Lyon

Sur le côté nord de la cour du temple d'Opet se trouvent les vestiges d'une stèle de granit noir dont H. Chevrier rapporte le dégagement lors de la campagne 1947-1948' ${ }^{\text {. Le }}$ monument est aujourd'hui très abîmé et le texte n'est presque plus visible, à l'exception de quelques bribes aux angles (pl. I). Une photographie de la surface inscrite, prise peu après la découverte, a gardé la trace de l'état — bien plus complet — de l'inscription à ce moment (pl. IV) ${ }^{2}$, ce qui permet d'en dégager la teneur et d'en postuler la datation: il s'agit d'une dotation d'offrandes au bénéfice du culte d'Osiris Ounnefer et de la déesse Opet instituée à l'époque kouchito-saïte. Il est probable que le cintre de la stèle était occupé par une représentation du roi offrant le signe de la campagne ${ }^{3}$ à ces dieux mais rien n'en n'a été préservé. Les premières lignes du texte sont également perdues.

Texte (pl. V)

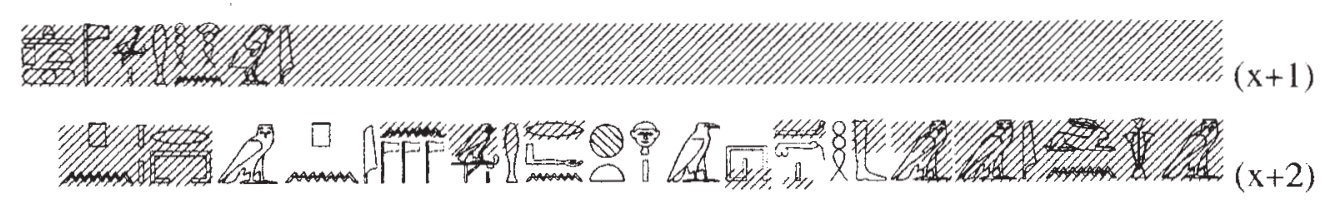

${ }^{1}$ H. Chevrier, «Rapport sur les travaux de Karnak, 1947-1948», ASAE 49 (1949), p. 5: «Une stèle de granit gris fut découverte contre le mur ouest de la cour. Elle est très abîmée par le salpêtre». M. Azim en mentionne encore la présence ( «À propos du pylône du temple d'Opet à Karnak», Karnak VIII [1987], p. 53, n. 10), ainsi que Cl. Traunecker, Les cryptes du temple d'Opet à Karnak, mémoire de l'EPHE, inédit, p. 40 et n. 251. Pas de mention dans le PM $\mathrm{II}^{2}$. Dimensions de la stèle: largeur hors tout, $1,00 \mathrm{~m}$; largeur du tableau plan: $0,86 \mathrm{~m}$; largeur entre les deux lignes verticales externes: 0,817 $\mathrm{m}$; hauteur hors tout: 1,00 m; épaisseur hors tout: $0,60 \mathrm{~m}$; hauteur des lignes de texte entre les traits horizontaux: en moyenne $5,38 \mathrm{~cm}$.

${ }^{2}$ Photo ancienne: CFEETK no 97851 et fiche CFEETK n ${ }^{\circ}$ 45122. Nous remercions N. Grimal et Fr. Larché, directeurs du Centre franco-égyptien d'étude des temples de Karnak, de nous avoir autorisés à publier ce document ainsi qu'O. Perdu et $\mathrm{D}$. Meeks pour leurs utiles suggestions.

3 «C'est là le geste habituel, chaque fois que le souverain agit en faveur des possessions meubles ou immeubles des dieux» (D. Meeks, «Une fondation memphite de Taharqa (Stèle du Caire JE 36861)», in Hommages Sauneron I [BdE 81] 1979, p. 222).

RdE 55 (2004) 


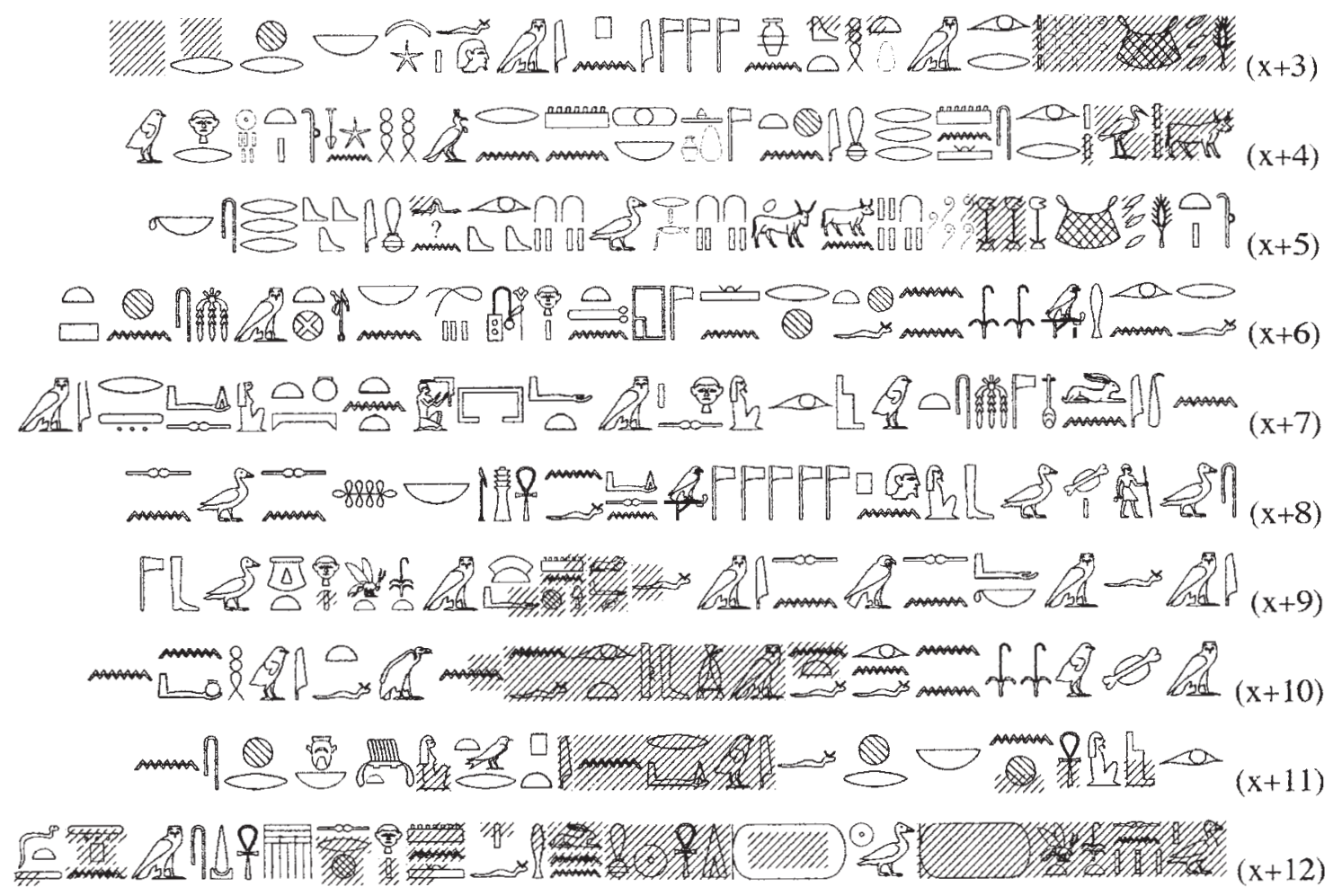

\section{Transcription:}

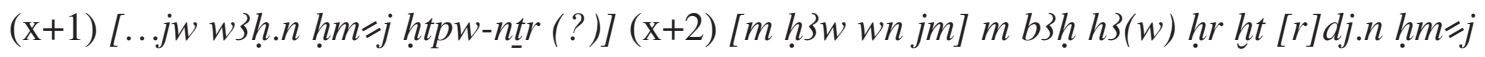
[n] ntrw jpn m [r(3)-pr pn (?)]

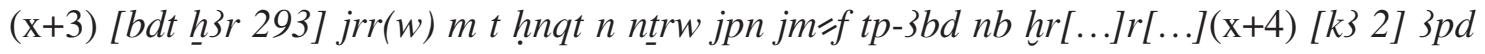
$2 \operatorname{jrr}(w)$

smn jrw mj ht $n$ ḥtpw-ntr nb mn $r$ nḥh

dmd sm3 $n$ rnpt 5 ḥr(y)w (x+5) rnpt: bdt ḩ3r: [3]516; k3 $n$ jdr(?): 24; r(3)-šd: 24;

jr wnm(t) nn (?) mjtt (?) jrw

sk (x+6) rf jr.n ḥm=j nn hft rh $n$ ḥwt-ntr ț $n$ ḥr sšw nb $n$ W $3 s t$

$m$ mshnt $(\mathrm{x}+7) n t$ Wn-nfr ms.tw Wsjr hr $=s m^{e} t$ jmnt nt Nwt

$d j=s r t 3 j m(\mathrm{x}+8)=s$ s3 smsw jw $w^{\circ} \mathrm{Gb} \operatorname{tp}(y) n$ ntrw 5

$d j=s n n=f^{e} n h \underline{d} \underline{d} d$ ws $n b$

$s 3=s n s 3=s n(\mathrm{x}+9) j m=f m k=s n s 3=s[n] j m=f$

$\left[j w^{e}\right] m n[h] h^{e}(w) m$ nswt bjty hrr nst $G b(\mathrm{x}+10) m$ jsw nn jr.n=f [n jt]=f [m $d b 3 w$ jr.n=f(?)] $n m w t=f$

jw ḥnk.n $(\mathrm{x}+11)$ Wsjr ${ }^{\mathrm{C}} \mathrm{h} h \mathrm{nb}$ hr=f [jw rdj.n Jpt]-wrt 3wt-jb nb hr=s $n(\mathrm{x}+12)[s 3=s n$

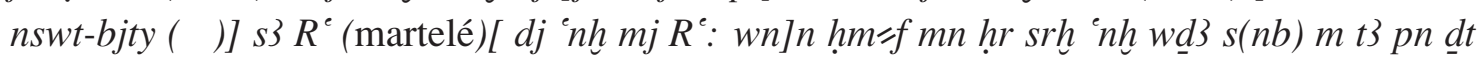




\section{Traduction}

$(\mathrm{x}+1)$ [... Ma Majesté a ordonné que soit instituée une offrande (?)] $(\mathrm{x}+2)$ [en accroissement de ce qui existait] auparavant ${ }^{\mathbf{a}}$, versée en sus des biens ${ }^{\mathbf{b}}$ que ma Majesté a donnés à ces divinités dans [ce sanctuaire (?) ${ }^{\mathrm{c}}$ ]: $(\mathrm{x}+3)$ [les 293 sacs de blé] qui conviennent ${ }^{\mathrm{d}}$, constituant l'offrande de pain-bière pour ces dieux qui s'y trouvent, mensuellement, [...], $(\mathrm{x}+4)$ les deux bovidés et les deux volailles qui conviennent ${ }^{\mathrm{e}}$, et que ceux-ci soient établis définitivement comme des biens (dévolus) à toute offrande divine, en étant fixés à jamais. Somme totale ${ }^{\mathbf{f}}$ pour l'année et les cinq jours épagomènes $(\mathrm{x}+5)$ : sacs de blé $\mathrm{g}$ : [3]516 ${ }^{\mathbf{h}}$, taurillons de génisse (?) ${ }^{\mathbf{i}}: 24$, oies-r(3) engraissées ${ }^{\mathbf{j}}: 24$, ce qui fait ${ }^{\mathbf{k}}$ de la nourriture ${ }^{\mathbf{l}}$ en quantité incomparable $(?)^{\mathbf{m}}$.

Or, $(\mathrm{x}+6)$ ma Majesté a fait cela en conformité avec les listes ${ }^{\mathbf{n}}$ de ce temple qui sont sur toutes les inscriptions de Thèbes dans le siège de naissance $(x+7)$ d'Ounnefer ${ }^{0}$ sur lequel Osiris a été enfanté, dans la chambre cachée de Nout; elle y a mis au monde ${ }^{\mathbf{p}}(\mathrm{x}+8)$ le fils aîné, l'héritier de Geb, le premier des cinq dieux ${ }^{\text {. }}$

Qu'ils (= Osiris et Nout) lui donnent toute vie, toute stabilité, toute force. En lui, c'est leur fils qu'ils protègent; en lui, c'est leur fils (Horus) qu'il gardent ${ }^{\mathrm{r}}(\mathrm{x}+9)$, l'héritier bienfaisant ${ }^{\mathrm{s}}$ apparu couronné en roi de Haute et Basse Égypte sur le trône de Geb, $(\mathrm{x}+10)$ en récompense de ce qu'il (= le roi) a fait [pour] son [père et en rétribution de ce qu'il a fait] pour sa mère ${ }^{t}$. Osiris a offert $(x+11)$ toute vie venant de lui [tandis qu'I]pet-ouret[a donné] toute joie venant d'elle au bénéfice de $(\mathrm{x}+12)$ [leur fils u, le roi de Haute et Basse Égypte ( )], fils de Rê (martelé ) ${ }^{\mathrm{v}}$ [gratifié de vie comme Rê. Puisse] Sa Majesté être établie sur le trône-serekh ${ }^{\mathbf{w}}$ - qu'il vive, soit prospère et en bonne santé — dans ce pays éternellement ${ }^{\mathbf{x}}$.»

Notes:

(a) $[m h 3$ h $w n$ jm] $m$ b3h : Les premiers signes conservés semblent bien correspondre à la fin de cette formule bien connue et que l'on retrouve, pour cette période, écrite ainsi: I Zeit des Apries», ZÄS 72 (1936), p. 41, col. 7 ); voir encore la stèle Louvre A 93, col. 5 (époque Amasis), publiée par E. Jelinkova-Reymond, ASAE 54 (1957), p. 276.

(b) ht est une désignation des biens, des possessions d'un temple. Cf. D. Meeks, Hommages Sauneron I (BdE 81), 1979, p. 234, n. (6) et 235, n. (16). Le mot se retrouve infra 1. x+4. Il doit s'agir du tour que l'on rencontre dans un passage parallèle du «texte de la jeunesse» de Thoutmosis III:

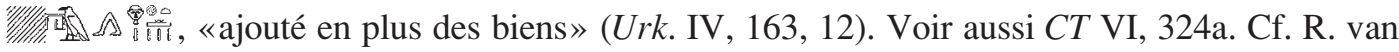
der Molen, A Hieroglyphic Dictionary of Egyptian Coffin Texts (PdÄ 15), 2000, p. 294.

(c) ntrw jpn m [r(3)-pr pn]: La restitution $r(3)-p r$ «sanctuaire» est suggérée par le groupe parallèle ntrw jpn jm=f «ces dieux qui s'y trouvent» de la ligne suivante $(\mathrm{x}+3)$ où $j m=f$ «y (= en lui)» renvoie nécessairement à un mot masculin désignant un lieu où résident des divinités. 
(d) $\operatorname{jrr}(w)$ : participe imperfectif passif avec ici une idée d'obligation (G. Lefebvre, Grammaire, §434, 436-37 et A.H. Gardiner, E.G. §369), «à faire, qu'il convient de faire». Pour les graphies à l'époque saïte, voir P. Der Manuelian, Living in the Past. Studies in Archaism of the Egyptian Twenty-sixth Dynasty, Kegan Paul International, 1993, p. 122.

(e) bdt ḩ3r 293 (...) hr[...] $r[\ldots]$ [k3 2] 3pd $2 \operatorname{jrr}(w)$ : On peut être certain de la restitution des volailles et de leur nombre. La restitution des deux taureaux et des 293 sacs de blé se déduit des chiffres donnés à la $1 . \mathrm{x}+5$ (voir infra $\mathrm{n}$. h) où le total annuel est récapitulé. Les pattes d'un taureau semblent visibles au début de la $1 . \mathrm{x}+4$. $h r$ pourrait avoir ici le sens de «avec» (D. Meeks, Alex I, 77.3140). Comme à la ligne précédente, $\operatorname{jrr}(w)$ a été compris comme un participe passif «à faire, qu'il convient de faire».

(f) dmd sm3: «somme totale». Sur la graphie $\mathrm{k}$, voir P. Posener-Krieger, Les archives du temple funéraire de Neferirkarê-Kakaï (BdE 65), 1976, I, p. 216-218; R. Caminos, The Chronicle of Prince Osorkon (AnOr 37), 1958, p. 62 et p. 65, n. 1; stèle de l'adoption de Nitocris, entre autres 1. x+23, cf. id., JEA 50 (1964), pl. X (= PRIRS 1 (2002), doc. 1, p. 24). L'emploi de cette expression développée est aussi un trait archaïsant. Cf. M. Megally, Recherches sur l'économie, l'administration et la comptabilité égyptiennes à la XVIII dynastie (BdE 71), 1977, p. 48.

(g) bdt ḩ $3 r$ : La mesure $\underline{h} 3 r$ «sac» s'écrit plus couramment $f$, mais le grand texte d'Amenemhat II trouvé au temple de Ptah à Memphis donne plusieurs exemples de notre graphie détaillée, voir H. Altenmüller, A.M. Moussa, «Die Inschrift Amenemhets II. aus dem Ptah-Tempel von Memphis. Ein Vorbericht», SAK 18 (1991), p. 44 et, par exemple 1. 12.

(h) Pour les centaines, 300 sont sûrs et il semble bien y avoir les vestiges de deux supplémentaires. Il n'y a pas de traces qui puissent correspondre à d'autres centaines qui viendraient s'ajouter à celles-ci, et ces cinq sont donc un maximum. En revanche, il y a de la place pour deux autres signes 1 (au plus), ce qui permet de supposer qu'il y avait à l'origine le nombre 3516. Cette restitution est corroborée par le fait qu'il s'agit d'un multiple de 12 (293 fois 12), comme les 24 oies et 24 taureaux de la suite de la liste, ce qui permet une répartition des biens sur les douze mois de l'année.

(i) Le premier bovidé est nettement distinct de la vache qui le suit et il faut y reconnaitre soit un veau déjà pourvu de cornes - un taurillon—, soit un taureau. Le groupe _ lu, sous toute réserve, $k 3 n j d t$ (voire $k 3 n j h t$ ou $k 3 n h m t$ ), est peut-être une retranscription de l'expression

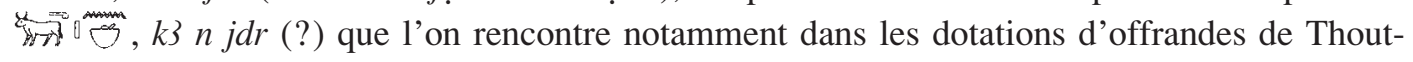
mosis III (par ex. Urk. IV, 195, 10 et 12) et dont le dictionnaire de Berlin fait un «taureau de troupeau» (?) (Wb. V, 98, 1 et I, 154, 14-15). Voir aussi W. Helck, Materialen zur Wirtschaftgeschichte des Neuen Reiches III, Wiesbaden, 1963, p. (481). Il y a peut-être glissement ou confusion entre le mot $\circlearrowleft j d r$ «troupeau» et le mot $j d t$, «femelle», et plus particulièrement «vache», (voir Ph. Collombert, «Quelques précisions sur la lecture et la signification du mot $\uparrow \ominus », R d E 46$ (1995), p. 205-208).

(j) Pour la lecture $r(3)-\check{s} d$, voir of Relief Fragments in Museums and Private Collections», SAK 13 (2002), p. 108, fig. 1); $P\{$. clairement le signe-šd et non $m h$ ) dans l'inscription dédicatoire de l'Akhmenou (Urk. IV, 1256, 
11 = A.H. Gardiner, JEA 38 (1952), p. 12, n. 7 et pl. V, col. 27). Nous devons ces références à D. Meeks. Voir aussi les annales d'Osorkon: Reliefs and Inscriptions at Karnak. III. The Bubastite Portal (OIP 74), 1954, pl. 21, col. 15 = R.A. Caminos, The Chronicle of Prince Osorkon (AnOr 37), 1958, p. 102, §155 (政). La lecture r3-mh préconisée par H. Kees («Die Kopenhager Schenkungstele aus der Zeit des Apries», ZÄS 72 (1936), p. 41. p. 45) est donc obsolète.

(k) La formule $j r(w)$, variante de $j r . n$ «ce qui fait», est effectivement attendue; voir D. Meeks, $L e$ grand texte des donations d'Edfou (BdE 59), 1972, particulièrement p. 157, §2; R.A. Caminos, The Chronicle of Prince Osorkon (AnOr 37), 1958, §202.

(I) Le mot $\Lambda \perp$, doit être $w n m(t)$ «ce qui se mange, nourriture» (Wb. I, 321, 15; R. van der Molen, A Hieroglyphic Dictionary of Egyptian Coffin Texts (PdÄ 15), 2000, p. 94). L'acception «nourriture» conviendrait d'autant mieux que l'énumération qui précède — du grain, des bovins et des oies — n'est effectivement constituée que de biens comestibles.

(m) Ce passage résiste à notre compréhension. La formule en apparence la plus proche serait $n n$ mjtt jrw. Pour un exemple daté de la XXVI e dynastie, voir la stèle de Psammétique II de Tanis (P. Der Manuelian, Living in the Past. Studies in Archaism of the Egyptian Twenty-sixth Dynasty, Kegan Paul International, 1993, p. 368, pl. 8 et 18, et en entérinant la correction prônée à la n. 290 de la p. 371). Néanmoins, le groupe des trois collines/pains reste inexpliqué, bien qu'il soit clair qu'il y a un jeu avec la graphie de ce que nous lisons wnmt. Il pourrait s'agir d'un terme signifiant «offrandes», le déterminatif des trois pains entrant dans la graphie de plusieurs termes appartenant à cette catégorie (cf. A.H. Gardiner, Egyptian Grammar, $3^{\text {rd }}$ ed., Oxford, 1957, p. 532, X7). Notons qu'au vu des traces subsistantes, une lecture $s n$ au lieu de $n n$ est encore envisageable, qui conduirait à une transcription $j r w n m=s n ~ m j w n m(w) j r w$ «faisant qu'ils (= les dieux) se nourrissent conformément à leurs rations alimentaires respectives».

(n) Pour $r$ h signifiant «liste», voir D. Meeks, Le grand texte des donations d'Edfou (BdE 59), 1972, p. 54. Pour $r h$ utilisé pour les «listes de fêtes», cf. A. Grimm (ÄAT 15), 1994, p. 157, n. B (a).

(o) La graphie - - Un du morphème du génitif féminin $n t$ est attestée dans les Textes des Pyramides (PT 1703 dM). Cf. E. Edel, Altägyptische Grammatik, Rome, 1955-1964, §325. Elle relève ici encore d'une recherche d'archaïsme.

(p) Ce passage évoquant la naissance d'Osiris dans le temple thébain d'Opet fait écho aux inscriptions du temple ptolémaïque, qui situent là sa $m s h n t$, «son siège de naissance»:

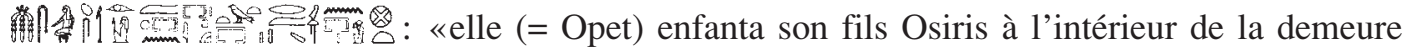
d'Opet la Grande, à l'ouest de la demeure de Khonsou» (Opet 171). Le temple lui-même est

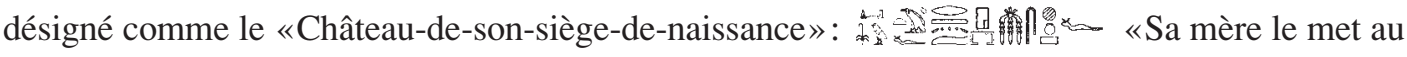
monde dans le Château-de-son-siège-de-naissance» (Opet 233). Sur la naissance du dieu à Thèbes, voir J. Yoyotte, «Une notice biographique du roi Osiris», BIFAO 77 (1977), p. 147 n. 3; S. Cauville, Le temple de Dendara. Les chapelles osiriennes. Commentaire (BdE 118), 1997, p. 237-238 et 267-268.

(q) $j w^{e} G b t p(y) n 5$ (djw) ntrw. Osiris est le premier des cinq dieux à naître pendant les cinq jours épagomènes (G. Poethke, LÄ I, col. 1231-32, s.v. «Epagomenen»; C. De Wit, Opet, 233 et

RdE 55 (2004) 
n. 251). H. De Meulenaere a recensé neuf autres attestations de la désignation $\operatorname{tp}(y) n 5$ (djw) ntrw pour ce dieu («La statue d'un vizir thébain. Philadelphia, University Museum E.16025», JEA 68 (1982), p. 142, n. 18). Références supplémentaires chez F.-R. Herbin, «La renaissance d'Osiris au temple d'Opet (p. Vatican inv. 38608)», RdE 54 (2003), p. 78 et 98.

(r) $s 3=s n s 3=s n j m=f m k=s n s 3=s[n] j m=f$ : Pour la valeur de $j m=f$ ici, voir E. Drioton, «Expressions prépositionnelles d'identité», ASAE 40 (1940), p.619-621 (référence que nous a communiquée D. Meeks). L'expression marque encore une recherche d'archaïsme. Notons ici également le jeu de mots entre le verbe $s 3$ «protéger» et le mot $s 3$ «fils». Pour la lecture $s 3$, «fils», du signe , var. voir H.W. Fairman, BIFAO 43 (1945), p. 106; P. Vernus, Athribis (BdE 74), 1978, p. 314, doc. 287. La répétition du thème, aussi bien dans son sens que dans sa forme, relève d'un glissement progressif du personnage divin (Osiris) vers le personnage du roi régnant, $\mathrm{du}$ mythique vers l'historique. Dans ce sens, l'emploi du signe du faucon (qui désigne implicitement le roi) dans le second terme est très significatif.

(s) Pour $j w^{e}$ mnh, qualifiant le roi, voir Urk. IV, 811, 14 (Thoutmosis III, monument d'Ellessiya).

(t) Pour ce topos de la rétribution divine des actions royales, voir par ex. l'une des stèles de Taharqa à Kawa où le lexique est identique (M.F.L. Macadam, The Temples of Kawa. I. The Inscriptions, Londres, 1949, pl. 6, col. 24): jr=f n=f jswy $m$ nn $m$ rdj $n=f$ ' $n h n h b$ hr=f, $\underline{d} \underline{d} t n b$ $h r=f$, ws $n b$ hr $=f$, snb $n b$ hr $r=f$, $3 w t-j b$ nb hr=f...

(u) Pour la restitution $n s 3=s n$, cf. la stèle de l'adoption de Nitocris, 1. x+ 14 (R.A. Caminos, JEA 50 (1964), pl. IX et p. $75=$ PRIRS 1 [2002], doc. 1, p. 22): $m$ 'nh ḥh, dd ḥh, w3s ḥh, snb 3wtjb $n b$ hr $=$ sn $n$ s3 $=s n m r=s n$...

(v) Pour la lecture du cartouche, voir infra le commentaire sur la datation du monument. Après le deuxième cartouche, on peut restituer $d j{ }^{c} n h \mathrm{hj} R^{e}$ ou ' $n h \underline{\underline{d}} \underline{\mathrm{d}}$.

(w) Pour le trône-sérekh, voir K.P. Kuhlmann, Der Thron im Alten Ägypten (ADAIK 10), 1977,

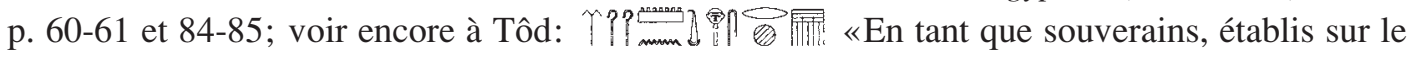
(trône)-serekh» (C. Thiers, Tôd II, 300, 2). Les restes du mot $m n$ sont encore identifiables sur notre stèle.

(x) Lecture assurée malgré la détérioration de la pierre. Comparer avec la stèle CGC 22183, 1. 28: 104 pays éternellement».

\section{Date du monument}

Des critères paléographiques et linguistiques autorisent à dater la stèle des $\mathrm{XXV}^{\mathrm{e}}-\mathrm{XXVI}^{\mathrm{e}}$ dynasties. Outre l'épigraphie extrêmement soignée et le caractère «classique» de la langue employée, on relèvera notamment les graphies archaïsantes de jrw $(1 \mathrm{x}+4$ et $\mathrm{x}+5)$, de h3r (1. $\mathrm{x}+5)$, de $n t-m$ H $(1 . \mathrm{x}+7)$, ainsi que les expressions et les tours teintés d'archaïsmes (ntrw ipn [1. $\mathrm{x}+2$ et $\mathrm{x}+3]$ s3 $=s n$ jm=f [1. $\mathrm{x}+8-9]$; dmd $\operatorname{sm} 3[1 . \mathrm{x}+4])^{4}$.

\footnotetext{
${ }^{4}$ Sur ces phénomènes, voir P. Der Manuelian, Living in the Past. Studies in Archaism of the Egyptian Twenty-sixth Dynasty, Kegan Paul International, 1993.
} 
Le cartouche de la dernière ligne du texte est très endommagé et la lecture du nom qui s'y trouvait est donc incertaine; il semble néanmoins ressortir de la photo ancienne que le nom a été martelé. À l'époque kouchito-saïte, de tels martelages sont attestés à l'encontre des pharaons éthiopiens puis de Néchao $\mathrm{II}^{5}$ et d'Amasis ${ }^{6}$. Des traces d'un signe horizontal en dépression dans le début du cartouche pourraient peut-être s'accorder avec la graphie que du nom de Néchao $\mathrm{II}^{7}$, voire à que ces lectures d'après des traces visibles sur une photographie ont d'hypothétique.

\section{Emplacement de la stèle et évolution architecturale du temple d'Opet}

Cette stèle datable des $\mathrm{XXV}^{\mathrm{e}}-\mathrm{XXVI}^{\mathrm{e}}$ dynasties apporte donc un élément nouveau sur l'histoire du temple d'Opet qui a subi de nombreuses transformations depuis le Nouvel Empire jusqu'à l'époque gréco-romaine ${ }^{8}$. L'existence d'un sanctuaire dédié à Opet-la-grande est attestée assez anciennement dans le secteur sud-ouest du temenos d'Amon, avec des vestiges qui remontent à Amenhotep II, voire à Thoutmosis III $^{9}$. Une statue remployée dans le dallage de l'angle sud-ouest de la seconde cour atteste l'existence d'un clergé d'Opet à l'époque ramesside $^{10}$. Le sanctuaire est ensuite réaménagé par Taharqa à la $\mathrm{XXV}^{\mathrm{e}}$ dynastie, ce dont témoignent de nombreux remplois. La construction du pylône est également attribuable à ce règne, essentiellement sur des critères architecturaux ${ }^{11}$. Malgré les nombreuses transformations du temple d'Opet à la $\mathrm{XXX}^{\mathrm{e}}$ dynastie et à l'époque ptolémaïque, la stèle, installée sur le parvis à proximité de ce pylône est probablement restée à son emplacement d'origine, les aménagements postérieurs ayant respecté en grande partie le parvis ancien.

\section{La fondation d'offrandes}

Malgré son caractère fragmentaire, le genre du texte peut être identifié sans hésitation: il s'agit d'un décret royal instituant une fondation d'offrandes pour un sanctuaire. Il

5 Cf. J. Yoyotte, Dictionnaire de la Bible, Supplément VI, 1960, col. 370-371; R.B. Gozzoli, «The Statue BM EA 37891 and the erasure of Necho II's names», JEA 86 (2000), p. 67-80; Egyptian Sculpture of the Late Period, Brooklyn, 1960, p. 50-51; H. de Meulenaere, Le surnom égyptien à la Basse Epoque, Istamboul, 1966, p. 28.

${ }^{6}$ H. de Meulenaere, $L \ddot{A}$ I, col. 182, s.v. «Amasis»; id., JEA 54 (1958), p. 184, n. 3.

7 GLdR IV, p. 87-88.

8 Sur la chronologie des différentes phases constructives, voir M. Azim, «À propos du pylône du temple d'Opet à Karnak», Karnak VIII (1987), p. 51-80. Pour un plan du parvis, voir M. Picker, J.-L. Bichet, Karnak VI (1980), fig. 21 en face de la page 58 , reproduit ici pl. XIII.

9 A. Varille, «La grande porte du temple d'Opet à Karnak», ASAE 53/1 (1953), p. 80, n. 1 et p. 112 n. 1 ; D. Meeks, «Ipet», LÄ III, 1980, col. 173-175; M. Azim «À propos du pylône du temple d'Opet à Karnak», Karnak VIII (1987), p. 53 et $n$. 15. Les fiches et les photos de la documentation de Karnak ne laissent pas de place au doute pour la lecture du nom de la déesse et la date des blocs.

${ }^{10} \mathrm{PM} \mathrm{II}^{2}, 251$; M. Azim, op. cit., p. 62, n. 74 (avec réf.). Pour le clergé du temple d’Opet, voir les références données par D. Meeks, «Ipet», LÄ III, 1980, col. 176 et n. 21; ajouter M. Abdelrahim, «Der Würfelhocker des Amunpropheten $H r$ (Kairo JE 38013)», in N. Kloth, K. Martin, E. Pardey (éd), Es werde niedergelegt als Schriftstück. Fs. H. Altenmüller, (BSAK 9), 2003, p. 1-6.

11 M. Azim, op. cit., p. 65-68. 
s'apparente ainsi, notamment, au décret de la fondation de Taharqa connu par la stèle Caire JE $36861^{12}$. Dans ce dernier texte, le roi, ayant pris acte de l'état de ruines dans lequel se trouvait le temple d'Amon-qui-préside-aux-temples à Memphis, décide de le faire reconstruire et le dote ensuite de mobilier ainsi que de possessions et de sources de revenus qui permettent la pérennité de son fonctionnement.

Certains termes apparaissant dans le texte de la stèle d'Opet appartiennent au vocabulaire technique spécifique à ce genre de documentation. Les termes $r d j$ «donner» et $h n k$ «offrir» sont les plus caractéristiques, l' «offrande» $(h n k)$ des dieux $(1 . x+10)$ devant probablement répondre au même terme appliqué à la donation du pharaon au début du texte. Le verbe smn «établir, instituer» est également fréquent dans les stèles thébaines relatives à des donations ${ }^{13}$. La formule $m n r n h h$ «établi à jamais» rappelle enfin les vœux d'éternité qui accompagnent les textes de cette nature ${ }^{14}$.

\section{Développement du culte osirien à l'époque kouchito-saïte}

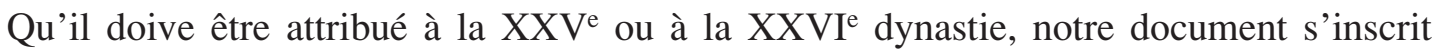
dans une période d'épanouissement des cultes d'Osiris à Thèbes. Pour la $\mathrm{XXV}^{\mathrm{e}}$ dynastie, les travaux de J. Leclant ont mis en lumière ce renouveau ${ }^{15}$. L'activité de Taharqa concernant précisément le temple d'Opet a laissé, on l'a évoqué, des traces sur le site: des architraves, un naos fragmentaire et probablement le pylône ${ }^{16}$. L'attention porté au culte osirien à l'époque saïte est attestée par de nombreuses constructions édifiées par les divines adoratrices Nitocris ${ }^{17}$ et Ankhnesneferibrê ${ }^{18}$, qui prolongent en cela l'intense activité architecturale vouée à Osiris durant la Troisième Période intermédiaire. Du règne de Néchao II date le réaménagement de la nécropole osirienne de la «Grande Place», au nord-est de Karnak $^{19}$. Un édifice voûté en briques cuites portant l'estampille de ce pharaon y avait été construit pour abriter les sépultures des figurines du dieu Osiris fabriquées annuellement

\footnotetext{
12 D. Meeks, Hommages Sauneron I, 1979, p. 221-259; id., «Les donations aux temples dans l'Égypte du Ier millénaire avant J.C.», in State und Temple, p. 607-608.

13 Ibid., p. 613 et n. 25. Pour smn employé dans le sens d' «établir durablement une offrande», voir par exemple l'une des stèles de Taharqa à Kawa dans M.F.L. Macadam, The Temples of Kawa. I. The Inscriptions, Londres, 1949, pl. 12, col. 25 (smn prt-hrw).

${ }^{14}$ H. Jacquet-Gordon, «A Donation Stela of Apries», RdE 24 (1972), p. 90; E. Graefe, M. Wassef, «Eine Fromme Stiftung für den Gott Osiris-der-seinen-Anhänger-in-der-Unterwelt-rettet aus dem Jahre 21 des Taharqa $(670$ v. Chr.)», MDAIK 35 (1979), p. 109, n. (h); A. Leahy, «Two Donation Stelae of Necho II», RdE 34 (1982-3), p. 83, n. (o).

15 J. Leclant, Recherches sur les monuments thébains de la XXVe dynastie dite éthiopienne (BdE 36), 1965.

16 Ibid, p. 82 [20]; M. Azim «À propos du pylône du temple d’Opet à Karnak», Karnak VIII (1987), p. 62, n. 75.

17 Voir L.A. Christophe, Karnak-Nord III (FIFAO 23), 1951, p. 113-128.

18 Voir L. Coulon, «Un aspect du culte osirien à Thèbes à l'époque saïte. La chapelle d'Osiris Ounnefer «maître des aliments»», Égypte. Afrique et Orient 28, (février 2003), p. 47-60.

${ }^{19}$ L. Coulon, F. Leclère, S. Marchand, "“Catacombes" osiriennes de Ptolémée IV à Karnak. Rapport préliminaire de la campagne de fouilles 1993», Karnak X (1995), p. 205-251; et en dernier lieu F. Leclère, «Fouilles dans le cimetière osirien de Karnak — travaux récents», BSFE 153 (2002), p. 24-44.
} 
lors des fêtes de Khoïak. Or, le temple d'Opet est également un lieu privilégié pour le déroulement des processions pendant ces fêtes, comme l'atteste le papyrus du Louvre N3176 (S), datant du début de l'époque romaine et détaillant les liturgies du mois de Khoïak à Karnak ${ }^{20}$.

On savait, par l'inscription de la statue stélophore de Bentehhor (Louvre A 83) que Néchao II avait, en l'an I de son règne, entrepris d'embellir Thèbes ${ }^{21}$ : le texte de la stèle y est conçu comme une Königsnovelle qui met en scène le roi prenant, devant ses courtisans, la décision de remédier à l'état lamentable dans lequel se trouvent les sanctuaires thébains. Si ce constat de ruine n'est pas avéré, le nombre des réalisations de Néchao dans la région concorde tout au moins avec cette proclamation ${ }^{22}$. S'il devait être assigné à ce règne, notre document pourrait apporter une confirmation à ces témoignages, le texte de refondation impliquant naturellement le déclin antérieur de l'institution concernée.

L'importance grandissante du culte d'Osiris à Karnak à l'époque tardive coïncide avec le rôle accru du mythe osirien dans l'idéologie royale. Notre document en offre un témoignage significatif, en révélant l'attention accordée par un pharaon kouchito-saïte au temple de la naissance d'Osiris. En garantissant l'institution des offrandes par un décret officialisé sur une stèle particulièrement soignée, le souverain proclame par la même occasion son statut d'héritier du dieu Osiris auquel on attribue ici une prérogative généralement dévolue à Amon sur le territoire de Thèbes.

\section{Résumé / Abstract}

Publication d'une stèle du parvis du temple d'Opet à Karnak, aujourd'hui très endommagée, mais dont un cliché ancien a conservé l'état au moment de son exhumation. Elle relate l'établissement d'une dotation annuelle d'offrandes au bénéfice du culte d'Osiris Ounnefer et de la déesse Opet instituée à l'époque kouchitosaïte, sous le règne d'un pharaon dont le nom, probablement martelé, est perdu.

Publication of a stela in the forecourt of the Opet temple at Karnak, presently very damaged, but of which an old photograph has preserved the image of its good condition at the moment of the discovery. It records the institution of a yearly offering donation for the cult of Osiris Wennefer and of the goddess Opet in the Kushito-saite epoch, under the reign of a pharaoh whose name, probably chiselled out, is now lost.

\footnotetext{
${ }^{20}$ P. Barguet, Le papyrus N. 3176 (S) du Musée du Louvre (BdE 37), 1962, p. 22 et 34. Pour la date du papyrus, voir J. Osing, Hieratische Papyri aus Tebtynis I, 1998, p. 29, n. 71; J.Fr. Quack, RdE 49 (1998), p. 255. F.-R. Herbin (RdE 54 [2003], p. 74-76) propose de rattacher également les liturgies du temple d'Opet contenues dans le p. Vatican inv. 38608 aux fêtes de Khoiak.

21 J. Yoyotte in Dictionnaire de la Bible. Supplément VI, Paris, 1960, col. 366 et 368, fig. 609; O. Perdu, «Prologue à un corpus des stèles royales de la XXVI e dynastie», BSFE 105 (1986), p. 24-26 (où l'auteur résume le texte de cette statue inédite).

22 J. Yoyotte, loc. cit.; D.B. Redford, LÄ IV, 1982, col. 370, s.v. Necho II.
}

RdE 55 (2004) 


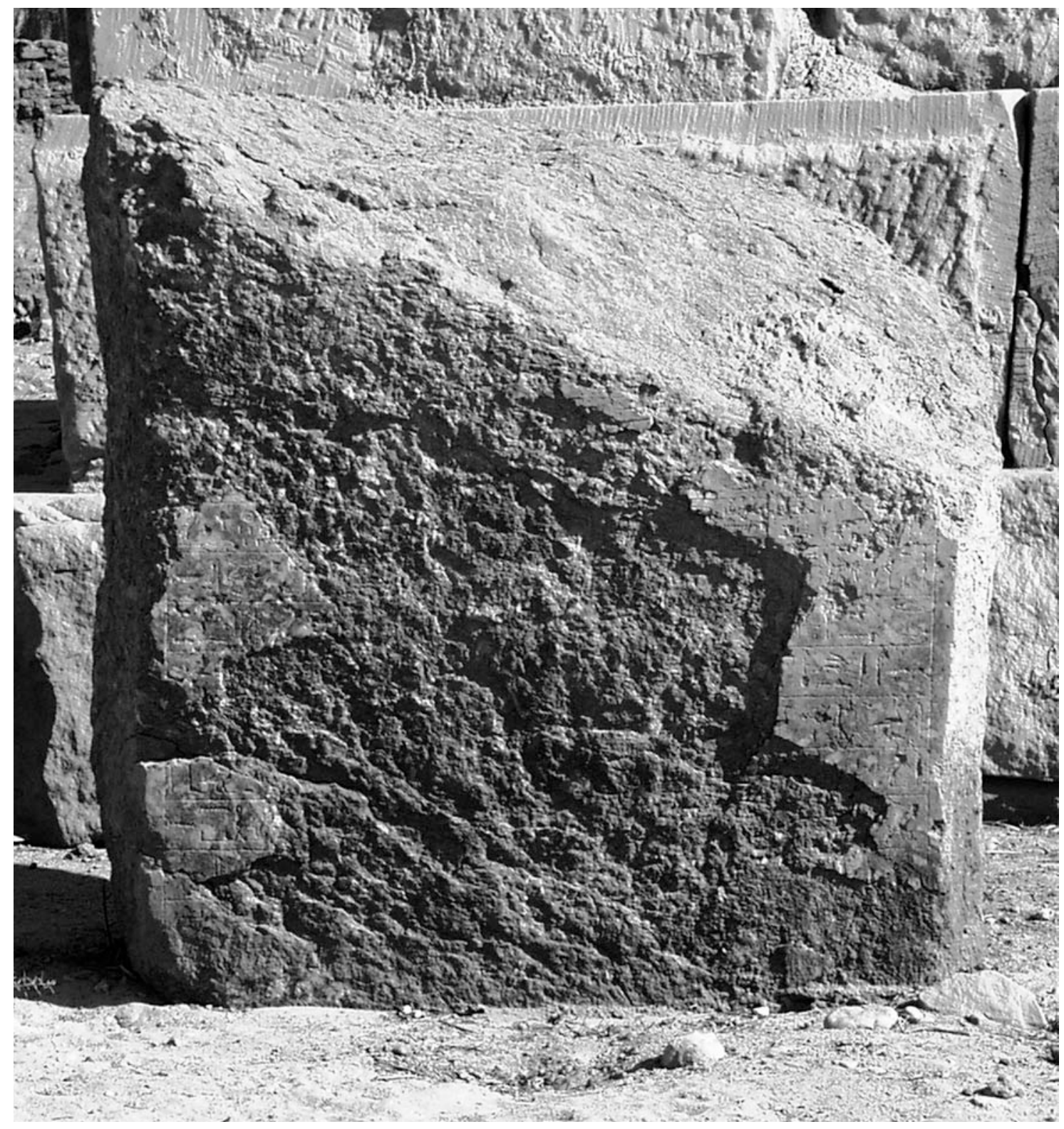

État actuel de la stèle (cl. L. Gabolde). 


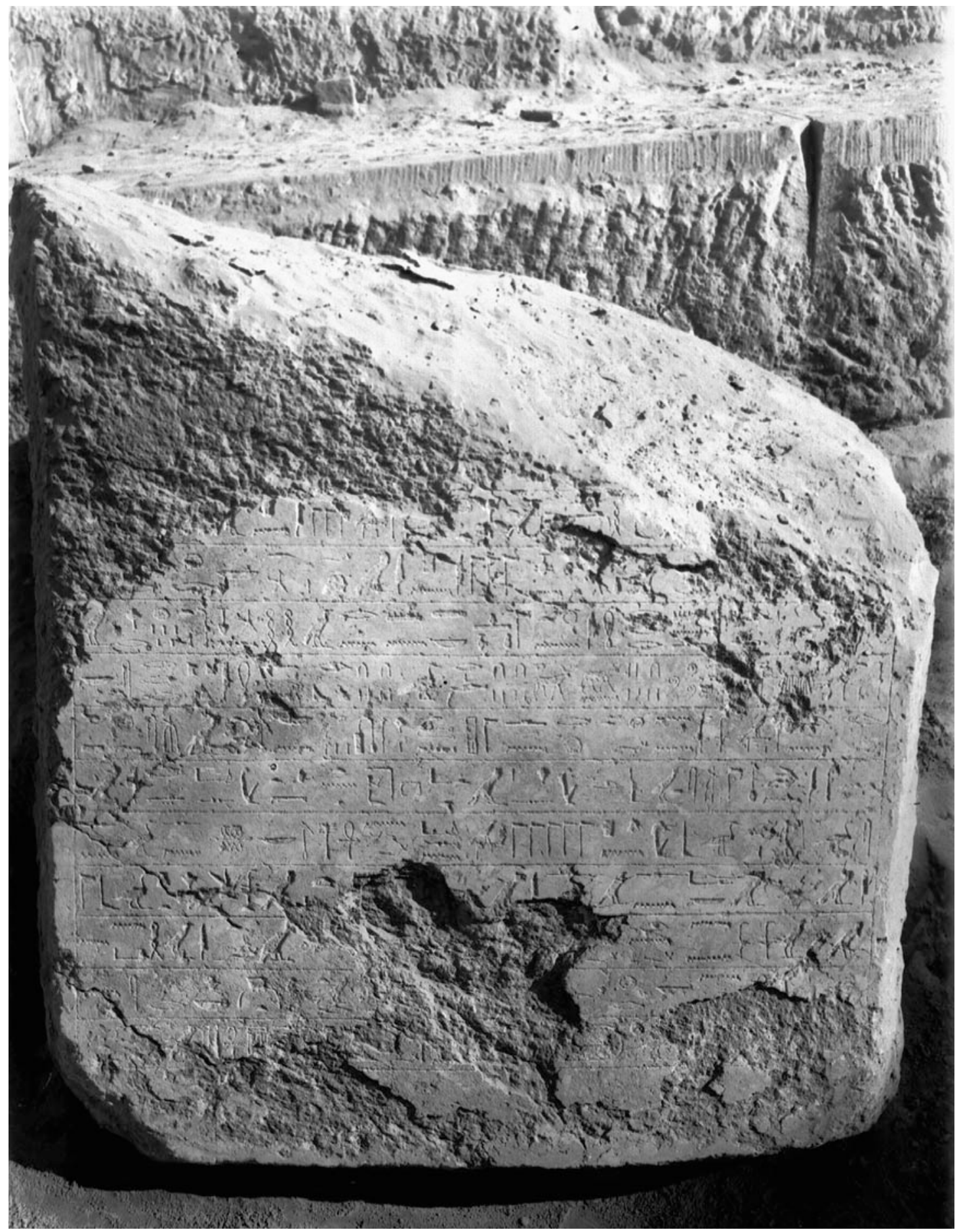

État de la stèle au moment de sa découverte (cl. fonds Chevrier/CFEETK, nº 97851).

L. Coulon - L. Gabolde, Une stèle sur le parvis du temple d'Opet à Karnak. 


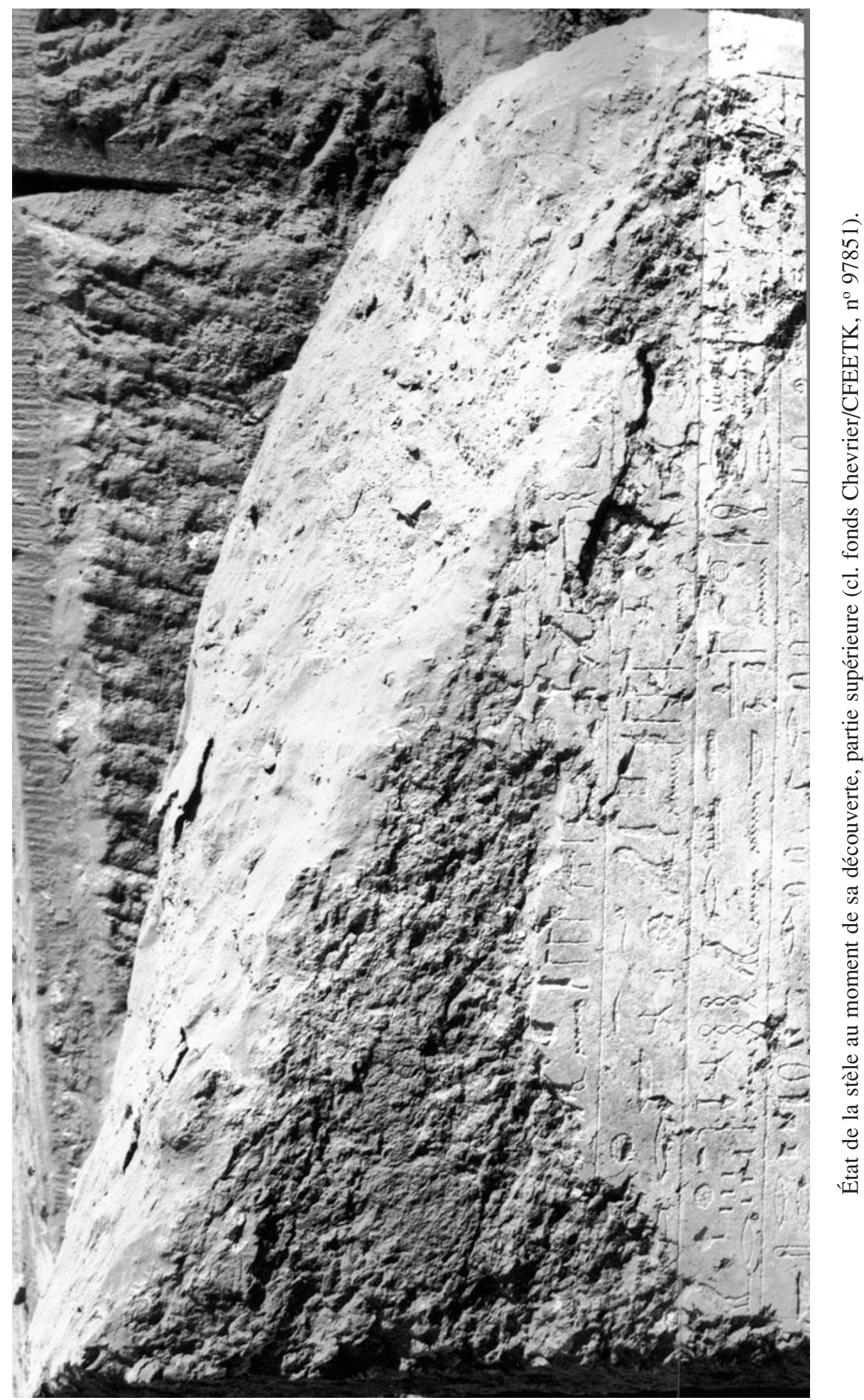

L. Coulon - L. Gabolde, Une stèle sur le parvis du temple d'Opet à Karnak. 


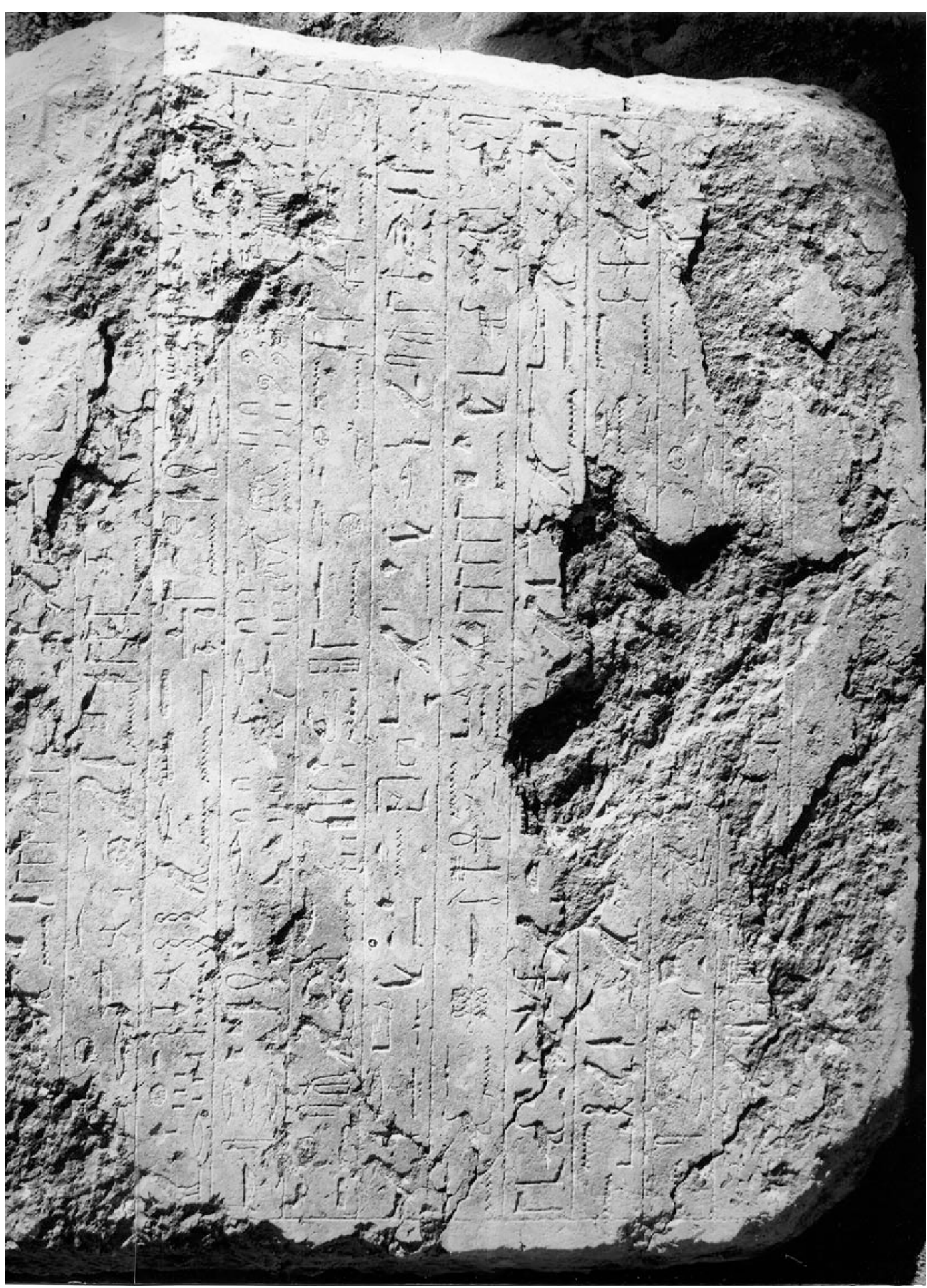




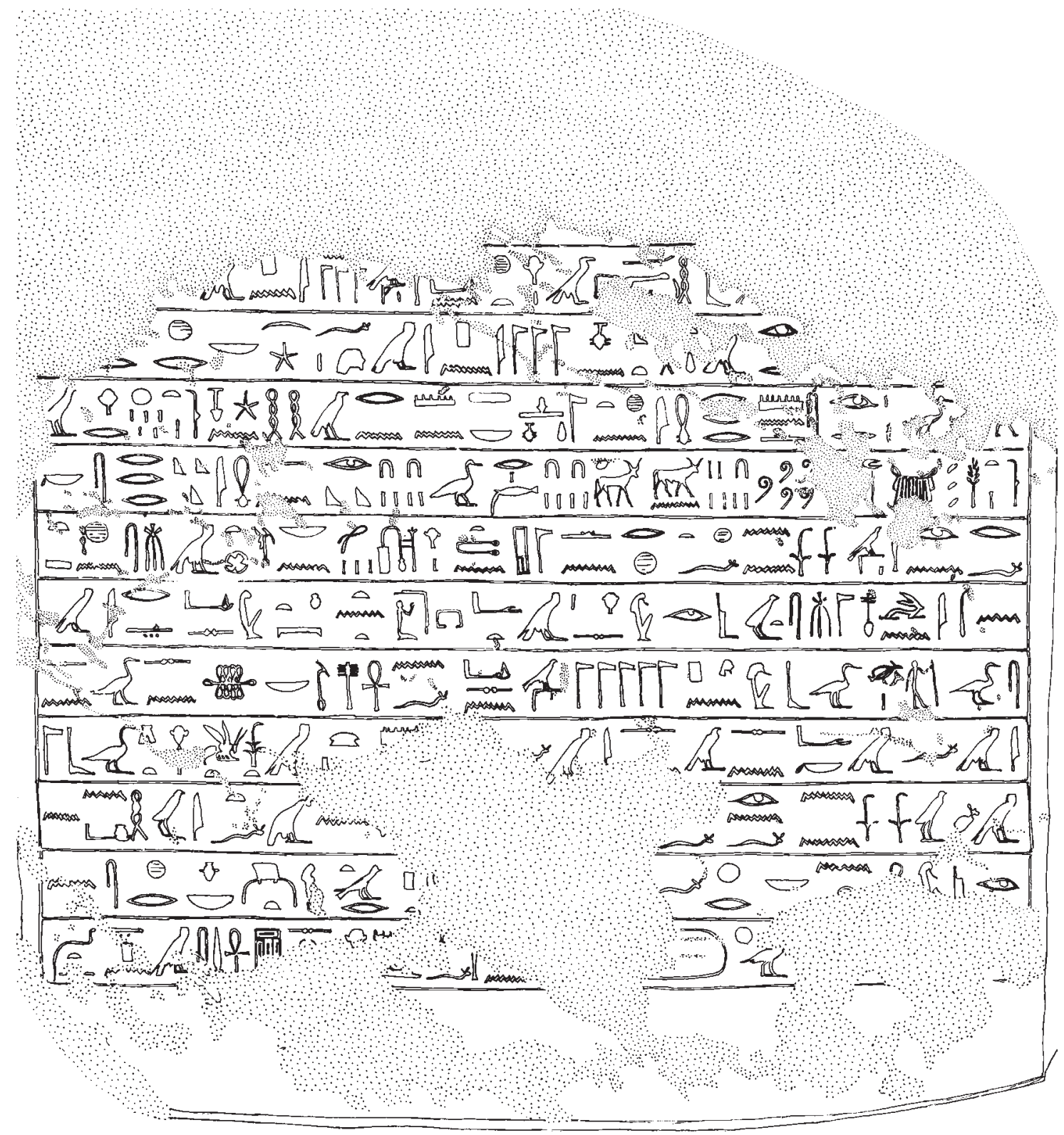

Fac-similé du texte.

L. Coulon - L. Gabolde, Une stèle sur le parvis du temple d'Opet à Karnak. 


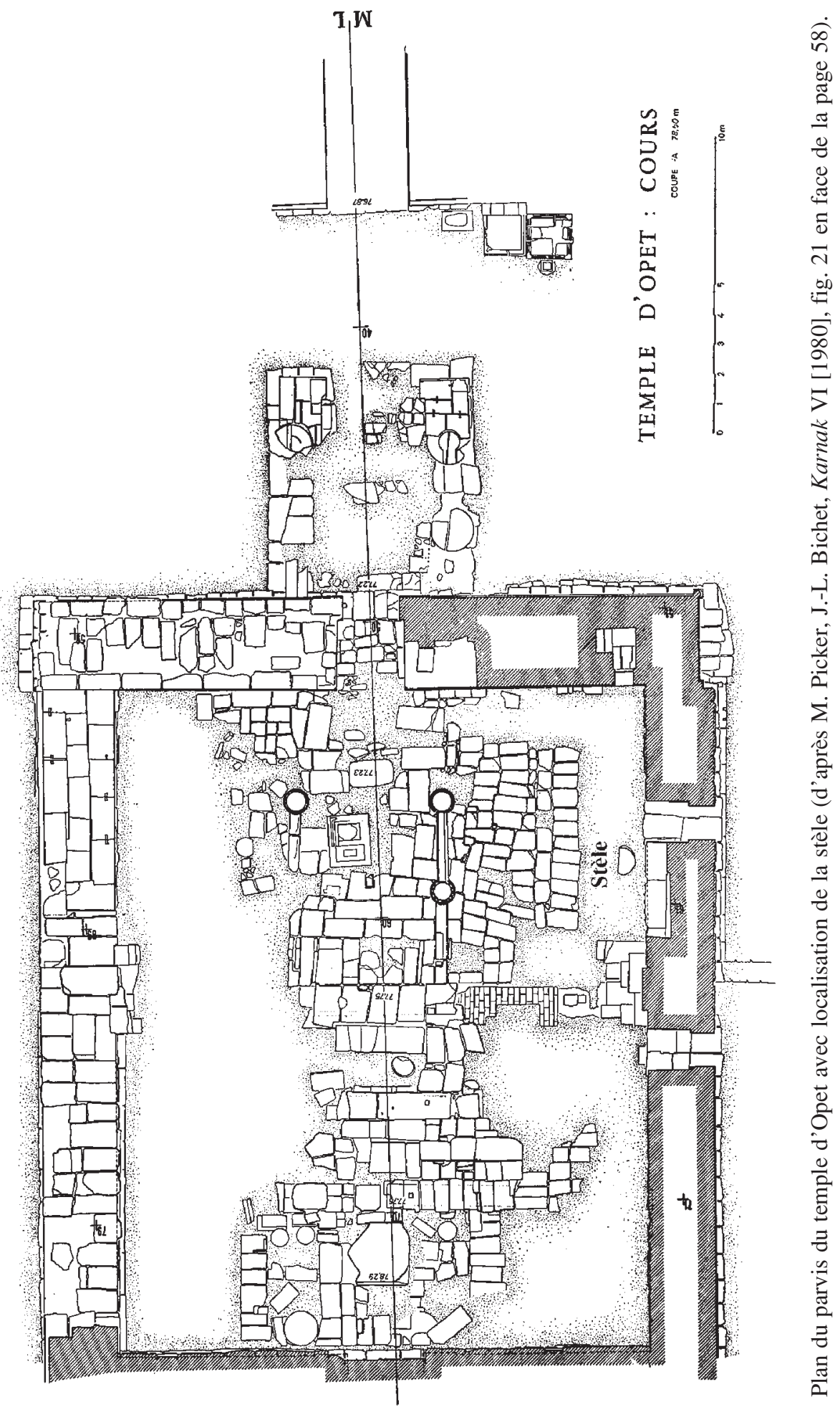

L. Coulon - L. Gabolde, Une stèle sur le parvis du temple d'Opet à Karnak. 